\title{
Fundamentos biomecánicos en el diseño de inmovilizador para rehabilitación de fracturas múltiples
}

\section{Bula Oyola, Ena Lucía}

Doctorando en Tecnologías para la Salud y el Bienestar, Universidad Politécnica de Valencia, España, Becario Desarrollo Profesoral, Universidad del Norte, Colombia, enbuoy@doctor.upv.es

\begin{abstract}
Bone pathologies involving the shoulder girdle are common and the rehabilitation is most frequently achieved through conservative treatments. We present the case of 20-year-old female patient with multiple trauma due to high-energy accident. Among the injuries, there was a displaced fracture of the diaphysis of the right clavicle, a displaced fracture of the left humerus, 11 fractures in 8 ribs (2 with displacement) and scapular dyskinesis.

Considering the instability of the thorax and the incompatibility between the fixation "8" (Watson-Jones) and the disposition of the displaced fragments, we designed a fracture immobilizer based on the functional analogy of the shoulder girdle. The external traction system developed emulates the action of the Pectoralis Major, Deltoid, Trapezius, Latissimus Dorsi, the upper area of the Oblique of the Abdomen and Serratus Anterior.
\end{abstract}

The continuous use of the product during six weeks allowed the consolidation of the fractures, restored the stability of the scapular girdle and the range of functional movement. The adequate traction of the fragments of the clavicle and its slight overlap contributed to attenuate the discontinuity in the shoulder.

The main achievements in the proposed design are: double traction effect; posterior fixation to keep the clavicle and shoulder blades in position under dynamic conditions and the possibility of applying pressure in a gradual and independent way in predetermined points. These principles could be applicable in the rehabilitation of other types of multiple fractures.

Keywords: rehabilitation, biomechanics, clinical experiences, exoskeletons, fractures, polytraumatism

\section{Resumen}

Las patologías óseas que afectan a la cintura escapular son comunes y en la mayoría de los casos su recuperación se obtiene a través de tratamiento conservador. Se presenta el caso de paciente femenino de 20 años con politraumatismo por accidente de alta energía. Entre las lesiones se 
encuentran: una fractura diafisiaria de clavícula derecha con desplazamiento, fractura diafisiaria del húmero izquierdo con desplazamiento, 11 fracturas en 8 costillas (2 con desplazamiento) y disquinesia escapular.

Teniendo en cuenta la inestabilidad torácica y la incompatibilidad entre la fijación en " 8 " (Watson-Jones) frente a la disposición de los fragmentos desplazados, se diseñó un inmovilizador basado en la analogía funcional de la cintura escapular y se estableció un sistema de tracciones externos que emulan la acción de los músculos Pectoral Mayor, Deltoides, Trapecio, Dorsal Ancho, la zona superior del Oblicuo Mayor del Abdomen y Serrato Anterior.

El uso continuo del producto durante seis semanas favoreció la consolidación de las fracturas, restituyó la estabilidad de la cintura escapular y el rango de movimiento funcional. La adecuada tracción de los fragmentos de la clavícula y su leve superposición contribuyó a atenuar la discontinuidad en el hombro.

Los principales beneficios en el diseño propuesto son: efecto de doble tracción, fijación posterior para mantener la clavícula y omoplatos en posición y bajo condiciones dinámicas, y la posibilidad de generar presión en forma gradual e independiente en puntos prefijados. Estos principios podrían ser aplicables en la rehabilitación de otro tipo de fracturas múltiples.

Palabras clave: rehabilitación; biomecánica; experiencias clínicas; exoesqueletos; fracturas; politraumatismo

\section{Introducción}

Las fracturas del tercio medio de la clavícula representan aproximadamente el 75\% de todas las fracturas de este hueso (Postacchini, Gumina, De Santis, \& Albo, 2002) y cerca de la mitad de ellas presentan desplazamiento (Robinson, 1998). Por su parte, las fracturas de costilla comprenden el 12\% de todas las fracturas observadas en pacientes (Barnea, Kashtan, Skornick, \& Werbin, 2002), la mayoría se deben a penetración directa o traumatismo cerrado en el tórax (Kuo \& Kim, 2019) y pueden causar contusiones pulmonares, hemotórax y/o neumotórax (Todd et al., 2006). El adecuado tratamiento para fracturas desplazadas es fuente de debate en la literatura actual, algunos estudios intentan establecer parámetros de medición (desplazamiento $>1,5$ o $2 \mathrm{~cm}$ ) que avalen la intervención quirúrgica y otros aluden a la revisión concreta de cada caso que derive en una decisión consciente. 
Con miras a aportar evidencia a la discusión actual acerca del tratamiento conservador de fracturas con desplazamiento, se presenta el caso de una paciente con politraumatismo tratada con éxito a partir del uso de un nuevo modelo de inmovilización inspirado en el sistema de tracción muscular del tronco y cintura escapular.

\section{Presentación de caso}

Paciente femenina de 20 años de edad, que al ir de pasajera en automóvil colisiona con otro vehículo resultando lesionada. Sufrió trauma de cráneo moderado con alteración de conciencia. Obnubilación. Trauma cerrado del tórax que ocasionó hemotórax y neumotórax de un $40 \%$. Fractura de la diáfisis de la clavícula derecha con desplazamiento de los fragmentos. Discinesia escapular. Fractura cerrada de húmero izquierdo cabalgada. Compromiso traumático del Nervio Radial (mano caída). Fractura del 3, 4, 5, 6, 7, 8, 9, 10 arco costal posterior izquierdo con desplazamiento de los fragmentos en algunos arcos costales (Fig. 1). Cirugía practicada: toracostomía de drenaje cerrado y acceso venoso central. Osteosíntesis de húmero, diáfisis con placas y Neurorrafia Radial.
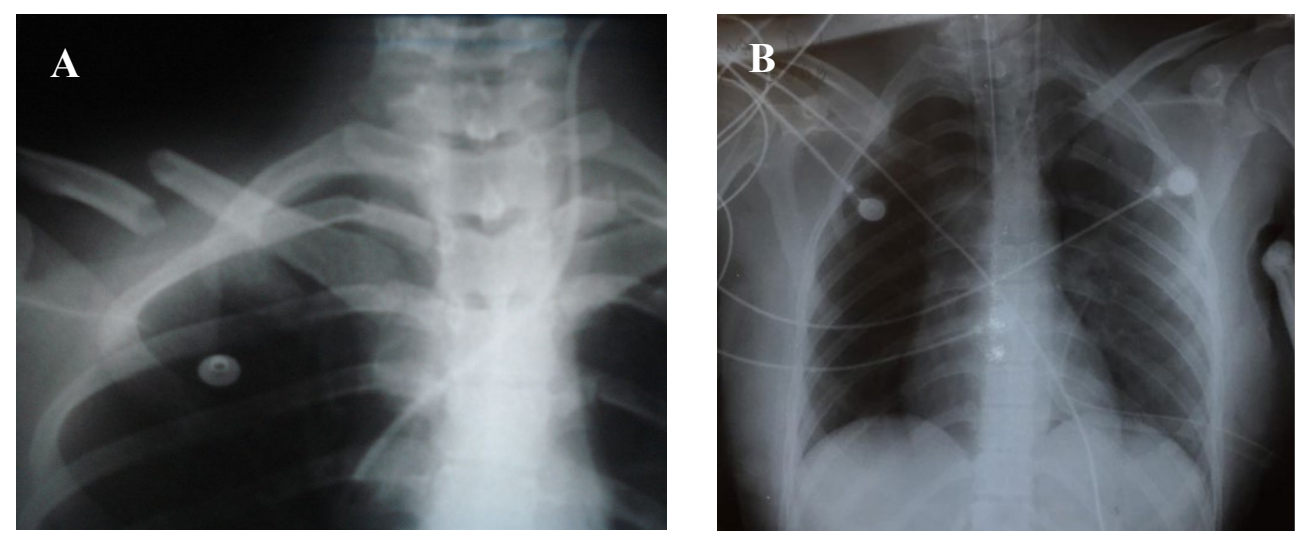

Fig. 1. Radiografías iniciales. (A) Fractura de clavícula. (B) Fracturas de arcos costales, Neumotórax y Hemotórax. Fuente: Clínica Cervantes (2007).

Al egresar de la unidad de cuidados intensivos le fue prescrita una órtesis radial y tratamiento conservador para la rehabilitación de las fracturas restantes, inmovilizador tipo Watson-Jones o "vendaje en 8" para la clavícula y faja para las costillas. 
El primer inmovilizador asignado usaba velcro como método de fijación que coincidía exactamente con la zona axilar, esto obligaba a la paciente a mantener sus brazos en abducción ante la incomodidad del material. En vista de la fluctuación de los fragmentos de la clavícula (síndrome de la tecla de piano) y sobrecarga en el húmero lesionado, optó por usar otro modelo del mismo tipo de inmovilización con fijación a través de hebillas. Con este ejemplar los brazos podían mantenerse en posición anatómica pero los vendajes no lograban presionar el fragmento elevado por acción del músculo esternocleidomastoideo. Posteriormente le recomedaron usar un cabestrillo sin mejores resultados.

El inmovilizador de costillas prescrito consistía en una faja elástica microperforada ajustable con velcro. El producto se situaba alrededor de la región costal inferior y contenía efectivamente los arcos costales inestables pero al menor movimiento se contraía y obligaba a ser fijado reiteradas veces. Esto incrementaba el dolor y la dificultad respiratoria.

Los inconvenientes presentados motivaron el diseño de una alternativa de inmovilización que favoreciera la consolidación de fracturas de clavícula y costillas de manera efectiva y confortable.

\subsection{Biomecánica y diseño}

El diseño del inmovilizador fue concebido por analogía funcional de las tensiones que actúan sobre la clavícula y costillas. La acción de los músculos Pectoral Mayor, Deltoides, Trapecio, Dorsal Ancho, la zona superior del Oblicuo Mayor del Abdomen y Serrato Anterior constituyen un mecanismo anatómico emulable para la estabilización de la cintura escapular y el tórax por medios ortésicos.
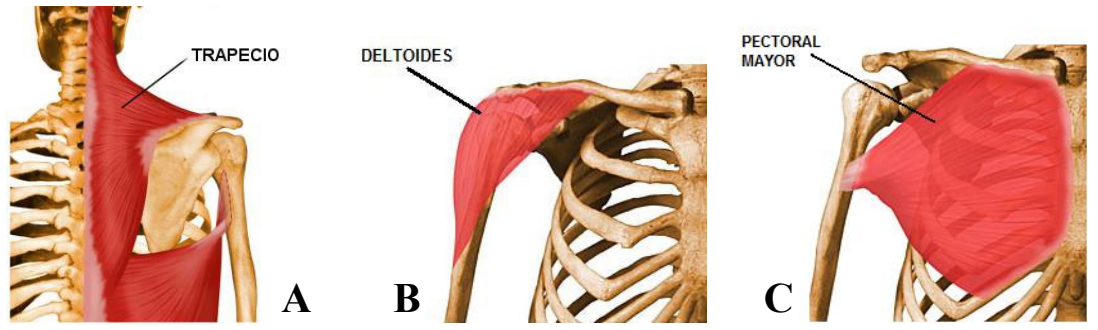

Fig. 2. Músculos Cintura Escapular. (A) Trapecio, (B) Deltoides y (C) Pectoral Mayor.

Fuente: Músculos.org $(n, d)$ 
Se tomó como referencia el Trapecio como estabilizador de la escápula durante los movimientos de la extremidad superior, que por medio de sus fibras horizontales medias tiran de la escápula hacia la columna. El Deltoides como eje de tracción de la zona distal de clavícula hacia el húmero y el Pectoral Mayor como eje de tracción de la zona proximal de la clavícula hacia el tronco (Fig. 2).
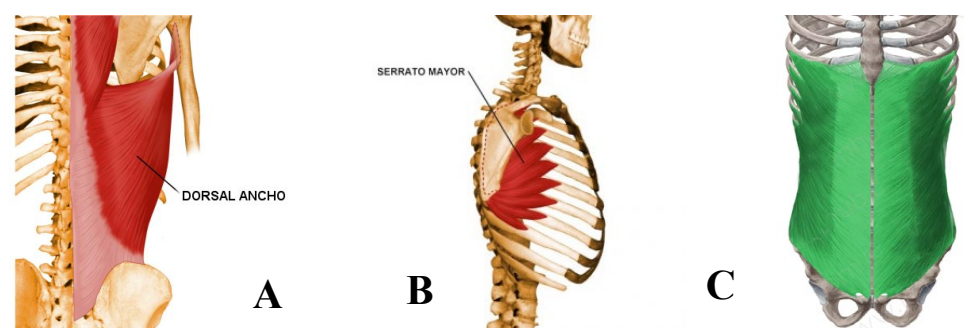

Fig. 3. Músculos de contención de arcos costales. (A) Dorsal Ancho, (B) Serrato Mayor y (C) Oblicuo Mayor del Abdomen. Fuente: Músculos.org $(n, d)$

Asimismo, se identificó la dirección de las tensiones anatómicas de los músculos que contribuyen a la protección de los arcos costales. El Dorsal Ancho que actúa como contención del tórax y el abdomen, el Serrato Anterior que cubre el área lateral del tórax intercalado entre las costillas y la escápula y que en sus fibras inferiores se encuentra con la unión costal del Oblicuo Mayor del Abdomen (Fig. 3).

El inmovilizador de tracción múltiple ó tracción cruzada es una unidad textil compuesta por una solapa que se ubica sobre las clavículas y escápulas ajustándose al tórax a través de dos franjas fijadoras de costillas unidas entre sí a partir de hebillas.
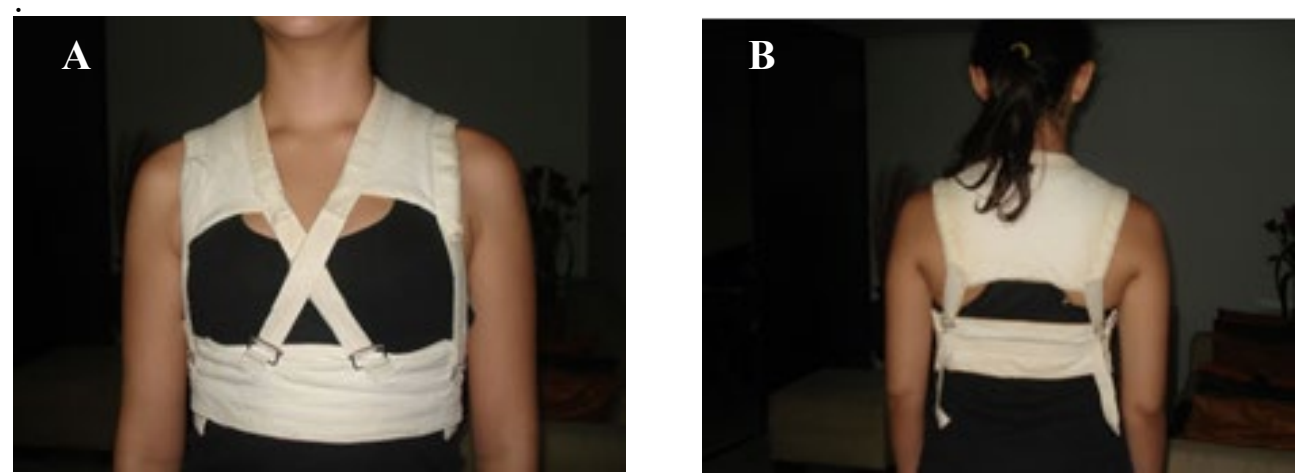

Fig. 4. Inmovilizador de tracción cruzada. (A) Vista frontal y (B) Vista posterior. 
Su configuración en "X" surge de la proyección de las fibras del músculo Pectoral Mayor como mecanismo que favorece el descenso del fragmento proximal de la clavícula, en procura de una posición más cercana a la alineación anatómica natural del hueso. Las franjas de sujeción dispuestas en cada uno de los extremos realizan presión perpendicular sobre el tercio distal y aseguran que la tensión sobre ambos fragmentos sea homogénea. En la zona posterior, la solapa se ajusta al corsé por medio de dos franjas cuyo fin es generar tracción opuesta a las tiras frontales y mantener el tronco erguido. La faja se ajusta mediante velcro y hebillas manteniendo los arcos costales en posición (Fig. 4).

\section{Resultados}

La paciente utilizó el inmovilizador diseñado durante 6 semanas. Durante el período de uso recibió sesiones de terapia respiratoria y posteriormente inició el programa de ejercicios para el fortalecimiento de la cintura escapular. A los 3 meses de seguimiento las fracturas habían consolidado.

\subsection{En función de su congruencia anatómica}

El abultamiento propio de la elevación del tercio proximal de la clavícula difícilmente recibe una presión adecuada que provoque su reducción a través del uso del inmovilizador en "8" (Fig. 5). El inmovilizador de tracción cruzada mantuvo una presión homogénea a lo largo del hueso. Sus tres puntos de presión con direcciones contrarias (hacia el esternón, la escápula y las costillas) restringieron en mayor medida el movimiento de los fragmentos y favorecieron la consolidación de la fractura (Fig.6).
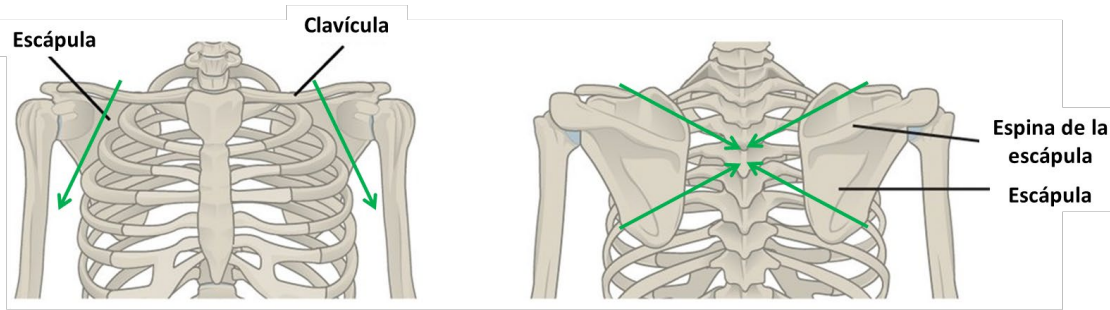

Fig. 5. Distribución de las tensiones sobre los fragmentos del "vendaje en 8 ". Fuente: OpenStax CNX CC 4.0 (2013). 
Bula, E.

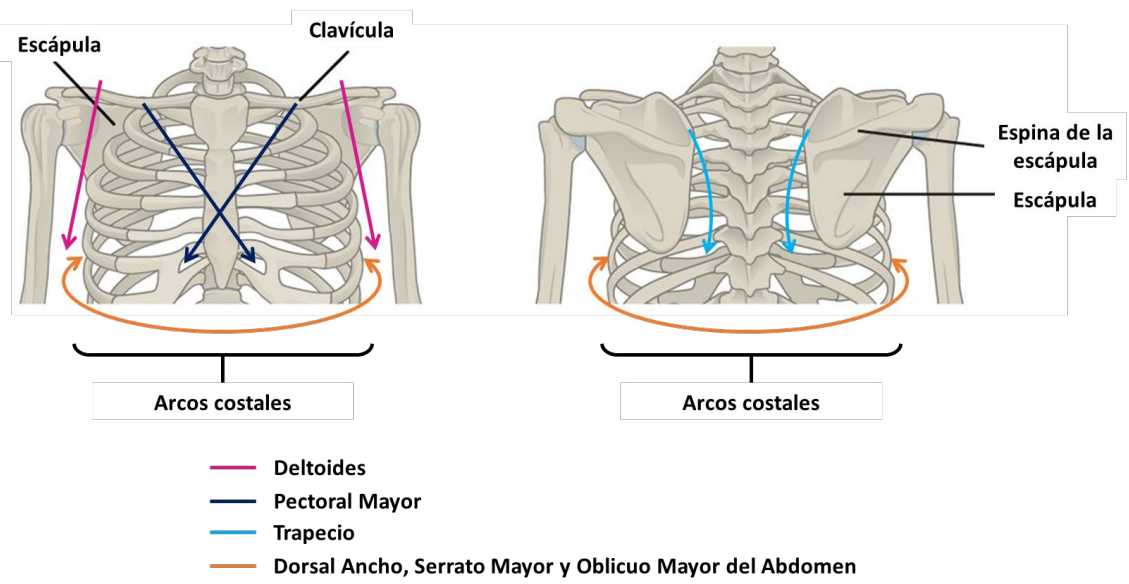

Fig. 6. Distribución de las tensiones sobre los fragmentos del inmovilizador de tracción cruzada. Fuente: OpenStax CNX CC 4.0 (2013).

La faja permitió contener los arcos costales de manera efectiva. El material semirrígido favoreció la estabilidad y su fijación a través de velcro y hebillas aseguró el ajuste, brindando una breve tolerancia para los movimientos respiratorios.

\subsection{En función de su uso}

El inmovilizador de tracción cruzada presenta una estructura similar a un corsé postural. La tensión que ejercen sus franjas posteriores hacia las escápulas favoreció la posición erguida del cuerpo y la reubicación de los fragmentos en dirección anteroposterior. Su configuración limitó los movimientos de elevación, protracción y retracción de la cintura escapular; así como la flexión y extensión del tronco sin interferir con otras zonas del cuerpo, proporcionando mayor comodidad e independencia.

\subsection{En función de la consolidación de las fracturas}

El proceso de rehabilitación se llevó a cabo con una considerable disminución de los síntomas dolor y alta correspondencia con los ejercicios respiratorios. Los fragmentos recibieron un correcto posicionamiento, a las 3 semanas se pudo notar que la evidente inestabilidad torácica había disminuido. A las 6 semanas de 
seguimiento se corroboró la consolidación total a través de exploración radiológica (Fig. 7).
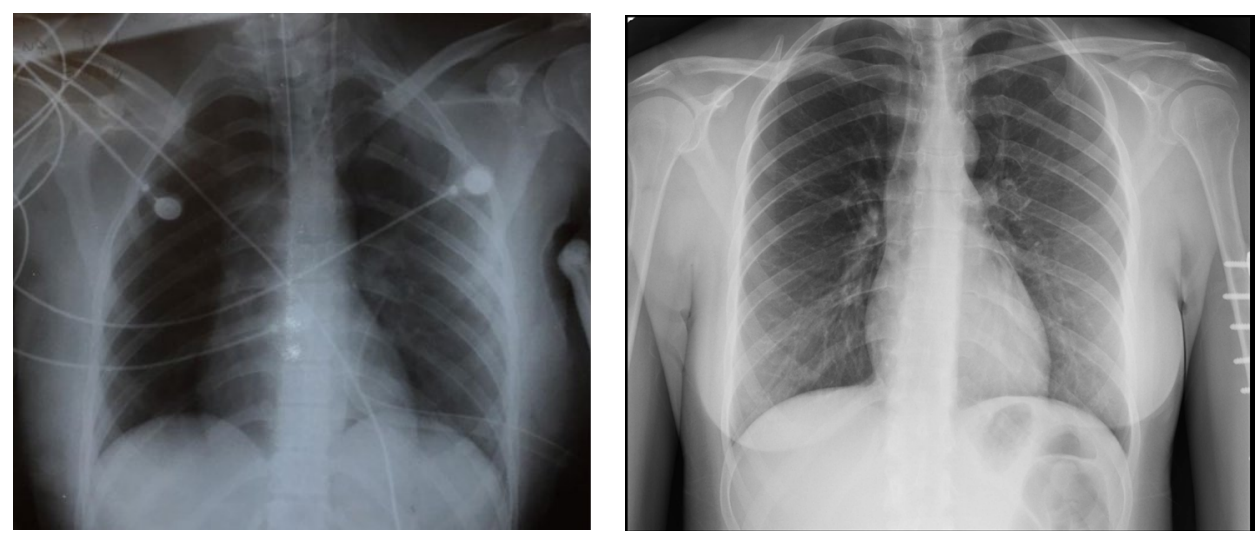

Fig. 7. . Evolución de las fracturas de costillas. (A) Radiografia inicial y (B) Radiografia posterior postratamiento con inmovilizador de tracción cruzada.
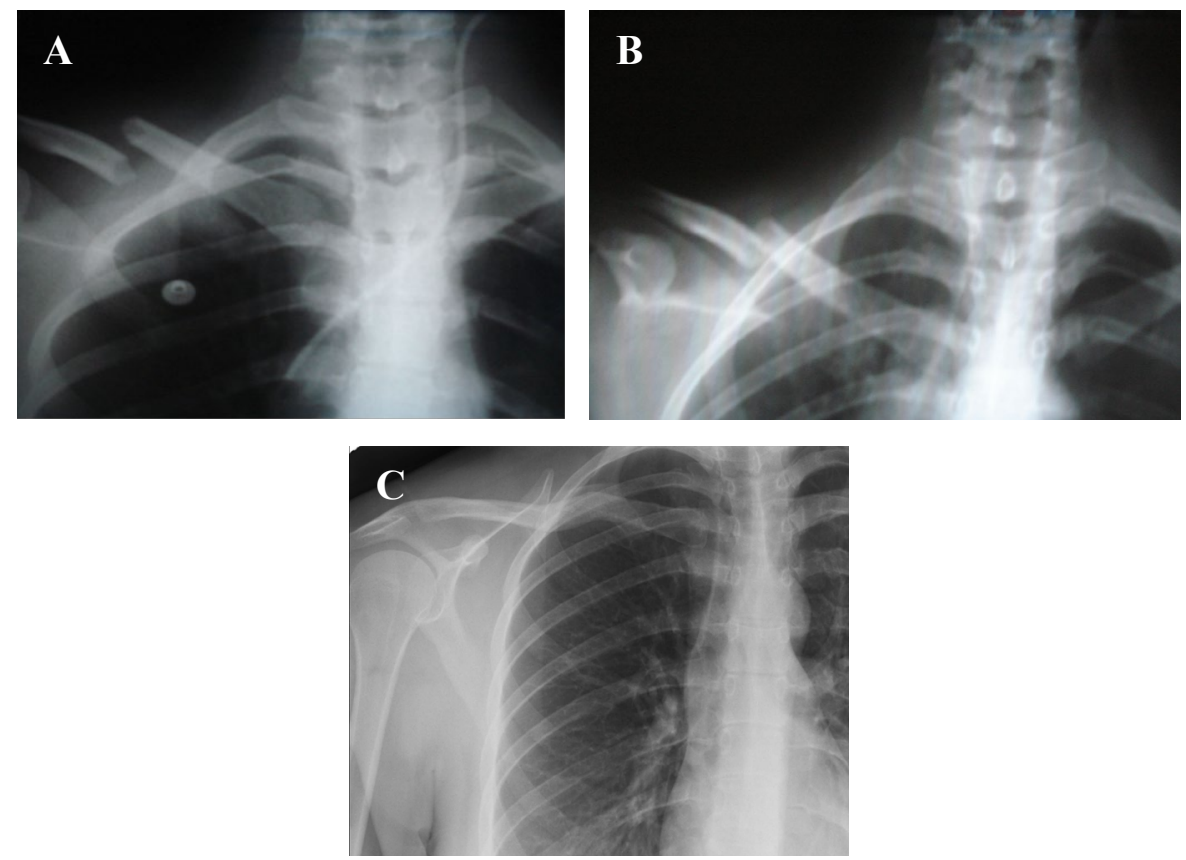

Fig. 8. Evolución de la fractura de clavícula. (A) Radiografia inicial y (B) Radiografía postratamiento seguimiento 3 meses (C) Radiografia posterior consolidación total. 
La rehabilitación de la clavícula se obtuvo en condiciones confortables. Al segundo día de uso, se identificó que el fragmento proximal había descendido completamente y se mantuvo en la misma posición durante las fases posteriores. Hubo una considerable disminución del dolor y funcionalidad del brazo derecho. A las 6 semanas de seguimiento se comprobó la consolidación con una notoria reducción del grado de desplazamiento (Fig. 8).

La reubicación temprana de los fragmentos logró minimizar considerablemente el nivel de acortamiento y volumen óseo. Rango de movilidad y fuerza conservados. La constitución física de la zona mantiene una apariencia cercana a la disposición natural del hueso, la alteración es inapreciable (Fig. 9).

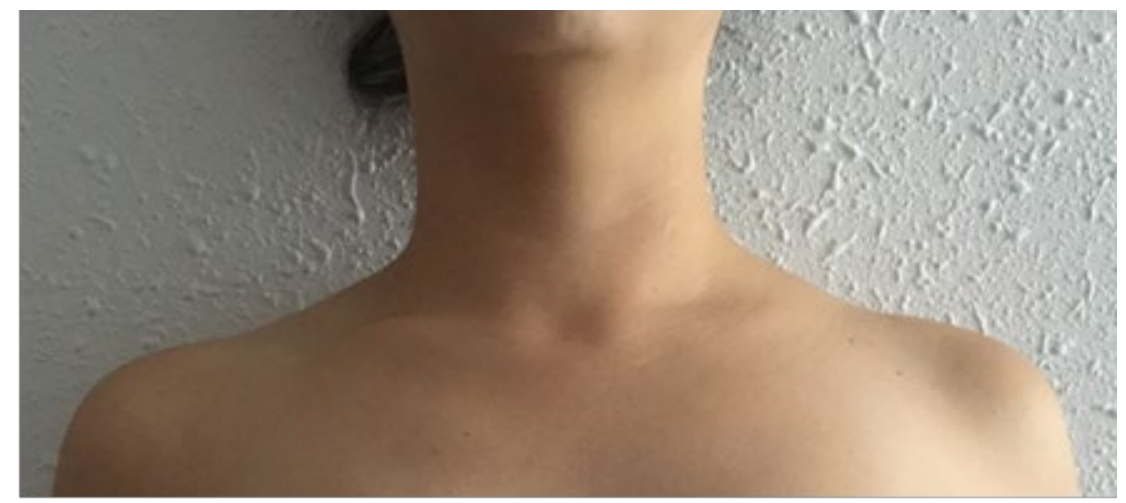

Fig. 9. Vista frontal de la apariencia física después de la consolidación de la fractura.

\section{Discusión}

Tradicionalmente, la mayoría de las fracturas de clavícula se han manejado de manera conservadora debido a las altas tasas de complicaciones del tratamiento quirúrgico (Rowe, 1960). Estudios realizados por Neer (1960) y Rowe (1960) resaltaron la efectividad del tratamiento conservador incluso en fracturas desplazadas, reportando una tasa de no unión menor del 1\%. Algunas revisiones recientes sugieren que la tasa asciente entre $15 \%$ y $20 \%$ (Canadian Orthopaedic Trauma Society, 2007; Hill, McGuire, \& Crosby, 1997; Nowak, Holgersson, \& Larsson, 2005; Zlowodzki et al., 2005) así como consolidación anómala que puede alterar la estructura de la cintura escapular, acortar la distancia hacia el hombro y desalinear las escápulas (Ristevski et al., 2013; Kim, Lee, Jang, Yeom, \& Banks, 2017; Rasyid, 2017). 
Existe controversia acerca de la efectividad del tratamiento conservador en presencia de desplazamiento. Frente a un aparente índice de alteraciones en la consolidación, estudios promueven la fijación quirúrgica primaria en pacientes que presentan deformidad visible o acortamiento de 1,5 o 2cm o más (Van Der Meijden, Gaskill, \& Millett, 2012; Wick, Müller, Kollig, \& Muhr, 2001). Otros abogan por la revisión individualizada de los casos (W. Kim \& McKee, 2008), teniendo en cuenta que en una clavícula muy larga (individuos altos) $2 \mathrm{~cm}$ puede tener menor efecto mecánico que la misma cantidad de acortamiento en una clavícula corta (R. Postacchini, Gumina, Farsetti, \& Postacchini, 2010).

El metaanálisis realizado por Lenza, Buchbinder, Johnston, Belloti, \& Faloppa (2013) destaca que no se encontraron pruebas que indiquen que la intervención quirúrgica tenga beneficios en términos de calidad de vida, y del efecto de la cirugía en el resultado estético debido a la calidad muy baja de las pruebas disponibles en siete ensayos. El tratamiento conservador reduce el riesgo de infección y cirugía secundaria debido a complicaciones, pero no hay certeza de la estimación del efecto y su precisión debido a la baja calidad de la evidencia.

Las fracturas de costillas múltiples son comúnmente tratadas de forma conservadora y a menudo requieren asistencia por medio de ventilación mecánica (Forward, Ollivere, Ng, Coughlin, \& Rollins, 2016). Ante las tasas de complicaciones ha habido un alza en la preferencia de métodos de fijación quirúrgica. Granetzny, Abd El-Aal, Emam, Shalaby, \& Boseila, (2005) identificaron mayores beneficios del tratamiento quirúrgico frente al conservador en el manejo de fracturas de costillas múltiples aisladas y dolorosas y Leinicke, Elmore, Freeman, \& Colditz (2013) concluyeron que la fijación quirúrgica del tórax inestable ofrece mejores resultados que el tratamiento conservador, reduce el porcentaje de mortalidad y el tiempo de asistencia hospitalaria.

En la revisión no se encontraron estudios de politraumatismo tratados de manera conservadora. El caso reportado expone resultados destacables en torno a mejora de la función muscular y respiratoria, tiempo y calidad en la consolidación de las fracturas y disminución del dolor. Se encontraron ventajas asociadas al uso, confort y satisfacción del paciente frente al tratamiento inicial (vendaje en 8 , cabestrillo y corsé elástico). El éxito de la intervención fue la visión integrada de todas las lesiones y sus afectaciones, incluyendo las no óseas. 
Es necesario validar la efectividad del tratamiento, la usabilidad y satisfacción en pacientes con fracturas múltiples en futuros estudios. De ser comprobada su utilidad y conveniencia, este método podría contribuir a minimizar los casos de pseudoartrosis o consolidación anómala y las alteraciones cinemáticas derivadas.

\section{Conclusión}

Se expuso un caso de fracturas múltiples con desplazamiento en paciente con politraumatismo severo tratada con un nuevo método de inmovilización. Los resultados indican que el principio de tracción multidireccional emulado del sistema muscular puede favorecer el proceso de consolidación conjunta de fracturas de clavícula y costillas, brindando excelentes resultados clínicos y físicos.

\section{Referencias}

BARNEA, Y., KASHTAN, H., SKORNICK, Y. y WERBIN, N. (2002). "Isolated rib fractures in elderly patients: mortality and morbidity". Canadian Journal of Surgery. Journal Canadien de Chirurgie, 45(1), 43-46.

CANADIAN ORTHOPAEDIC TRAUMA SOCIETY. (2007). "Nonoperative treatment compared with plate fixation of displaced midshaft clavicular fractures. A multicenter, randomized clinical trial". The Journal of Bone and Joint Surgery. American Volume, 89(1), 1-10.

FORWARD, D. P., OLliVERE, B. J., NG, J. W. G., COUGHLIN, T. A., y ROLLINS, K. E. (2016). "Current concepts in rib fracture fixation". Bone \& Joint $360,5(5), 2-7$.

GRANETZNY, A., ABD EL-AAL, M., EMAM, E., SHALABY, A. y BOSEILA, A. (2005). "Surgical versus conservative treatment of flail chest. Evaluation of the pulmonary status". Interactive CardioVascular and Thoracic Surgery, 4(6), 583587.

HILL, J. M., MCGUIRE, M. H. y CROSBY, L. A. (1997). "Closed treatment of displaced middle-third fractures of the clavicle gives poor results". The Journal of Bone and Joint Surgery, 79(4), 537-539.

KIM, D. S., LEE, D. W., JANG, Y. H., YEOM, J. S. y BANKS, S. A. (2017). "Effects of short malunion of the clavicle on in vivo scapular kinematics". Journal of Shoulder and Elbow Surgery, 26(9), e286-e292.

KIM, W. y MCKEE, M. D. (2008). "Management of Acute Clavicle Fractures". 
Orthopedic Clinics of North America, 39(4), 491-505.

KUO, K.. y KIM, A. M. (2019). Rib Fracture. StatPearls (Vol. 2011). Treasure Island: StatPearls Publishing.

LEINICKE, J. A., ELMORE, L., FREEMAN, B. D. y COLDITZ, G. A. (2013). Operative Management of Rib Fractures in the Setting of Flail Chest". Annals of Surgery, 258(6), 914-921.

LENZA, M., BUCHBINDER, R., JOHNSTON, R. V, BELLOTI, J. C. y FALOPPA, F. (2013). "Surgical versus conservative interventions for treating fractures of the middle third of the clavicle". Cochrane Database of Systematic Reviews, 6(9), CD009363.

NEER, C. S. (1960). "Nonunion of the clavicle". Journal of the American Medical Association, 172, 1006-1011.

NOWAK, J., HOLGERSSON, M. y LARSSON, S. (2005). "Sequelae from clavicular fractures are common: A prospective study of 222 patients". Acta Orthopaedica, 76(4), 496-502.

POSTACCHINI, F., GUMINA, S., DE SANTIS, P. y ALBO, F. (2002). "Epidemiology of clavicle fractures". Journal of Shoulder and Elbow Surgery, 11(5), 452-456.

POSTACCHINI, R., GUMINA, S., FARSETTI, P. y POSTACCHINI, F. (2010). "Long-term results of conservative management of midshaft clavicle fracture". International Orthopaedics, 34(5), 731-736.

RASYID, H. N. (2017). "Influence of Displaced Clavicle Fracture toward Scapular Motion". OALib, 04(05), 1-7.

Ristevski, B., HALl, J. A., PEARCE, D., POTTER, J., FARRUGiA, M. y MCKEE, M. D. (2013). "The radiographic quantification of scapular malalignment after malunion of displaced clavicular shaft fractures". Journal of Shoulder and Elbow Surgery, 22(2), 240-246.

ROBINSON, C. M. (1998). "Fractures of the clavicle in the adult. Epidemiology and classification". The Journal of Bone and Joint Surgery. British Volume, 80(3), 476484.

ROWE, C. R. (1960). "An atlas of anatomy and treatment of midclavicular fractures". Clinical Orthopaedics and Related Research, 58(10), 29-42.

TODD, S. R., MCNALLY, M. M., HOLCOMB, J. B., KOZAR, R. A., KAO, L. S., GONZALEZ, E. A. y MOORE, F. A. (2006). "A multidisciplinary clinical pathway decreases rib fracture-associated infectious morbidity and mortality in high-risk trauma patients". American Journal of Surgery. 
VAN DER MEIJDEN, O. A., GASKILL, T. R., y MILLETT, P. J. (2012). "Treatment of clavicle fractures: current concepts review". Journal of Shoulder and Elbow Surgery, 21(3), 423-429.

WICK, M., MÜLLER, E. J., KOLLIG, E., y MUHR, G. (2001). "Midshaft fractures of the clavicle with a shortening of more than $2 \mathrm{~cm}$ predispose to nonunion". Archives of Orthopaedic and Trauma Surgery, 121(4), 207-211.

ZLOWODZKI, M., ZELLE, B. A., COLE, P. A., JERAY, K., MCKEE, M. D., y EVIDENCE-BASED ORTHOPAEDIC TRAUMA WORKING GROUP. (2005). "Treatment of acute midshaft clavicle fractures: systematic review of 2144 fractures: on behalf of the Evidence-Based Orthopaedic Trauma Working Group". Journal of Orthopaedic Trauma, 19(7), 504-507.

\section{Imágenes}

MÚSCULOS.ORG (n,d). Músculo Deltoides. [imagen] Disponible en: https://www.musculos.org/musculo-deltoides.html [Acceso 2 Mayo de 2019].

MÚSCULOS.ORG (n,d). Músculo Dorsal Ancho. [imagen] Disponible en: https://www.musculos.org/musculo-dorsal-ancho.html [Acceso 2 Mayo de 2019].

MÚSCULOS.ORG (n,d). Músculo Oblicuo Mayor del Abdomen. [imagen] Disponible en: https://www.musculos.org/musculo-abdominales-oblicuo-mayorabdomen.html [Acceso 2 Mayo de 2019].

MÚSCULOS.ORG (n,d). Músculo Pectoral Mayor [imagen] Disponible en: https://www.musculos.org/musculo-pectoral-mayor.html [Acceso 2 Mayo de 2019].

MÚSCULOS.ORG (n,d). Músculo Serrato Mayor [imagen] Disponible en: https://www.musculos.org/musculo-serrato-mayor.html [Acceso 2 Mayo de 2019].

MÚSCULOS.ORG (n,d). Músculo Trapecio. [imagen] Disponible en: https://www.musculos.org/musculo-trapecio.html [Acceso 2 Mayo de 2019].

OPENSTAX CNX (2013). Cintura escapular. [imagen] Disponible en: https://cnx.org/resources/57d6e706ac983c0aa78022708939083d37027c4a/Figure 38 01_10.jpg [Acceso 8 Mayo de 2019]. CC 4.0 